Research Article

\title{
Crowd Sensing Urban Healthy Street Monitoring Based on Mobile Positioning System
}

\author{
Fang Jiang $\mathbb{D}^{1},{ }^{1}$ Yunjuan Sun, ${ }^{2}$ and Jianfeng Sha ${ }^{3}$ \\ ${ }^{1}$ Hubei University of Education, Wuhan 430205, Hubei, China \\ ${ }^{2}$ School of Fine Arts, Central South University for Nationalities, Wuhan 430205, Hubei, China \\ ${ }^{3}$ Wuhan Transportation Development Strategy Institute, Wuhan 430074, Hubei, China \\ Correspondence should be addressed to Fang Jiang; jiangfangjenny@hue.edu.cn
}

Received 29 June 2021; Revised 16 August 2021; Accepted 23 August 2021; Published 27 September 2021

Academic Editor: Sang-Bing Tsai

Copyright (C) 2021 Fang Jiang et al. This is an open access article distributed under the Creative Commons Attribution License, which permits unrestricted use, distribution, and reproduction in any medium, provided the original work is properly cited.

With the rapid development of social economy, the importance of ecological civilization is increasing day by day. The level of environmental monitoring will directly affect the control of total pollution sources and the evaluation of environmental quality. Crowd sensing is to use the group computing power of users with smart devices to quickly collect surrounding multidimensional data, analyze and calculate based on these massive perception data, and then dig out group behavior patterns and other regular information. In order to meet the basic requirements of environmental protection work put forward by the government in the new era, this paper proposes mobile positioning and crowd sensing technologies, explains the distribution map method and adaptive weighting data fusion related algorithms, and designs and develops a mobile positioning system based on mobile positioning. The system of crowd sensing of urban healthy street monitoring is designed, and then we use this PM2.5 monitoring system to monitor the relevant environmental data of a street in Shanghai and use normalization to process the experimental data. The experimental data and statistical results show that this performance of the system is good, and the accuracy of image PM2.5 monitoring reaches $90 \%-95 \%$. More than $90 \%$ of street residents are also very satisfied with this system, believing that it can play a role in supervising urban healthy street.

\section{Introduction}

1.1. Background. In recent years, China's urbanization and industrialization have accelerated, car ownership and urban construction have continued to grow, green space and water resources have gradually decreased, and PM2.5 air pollution has become more serious. In the cities of our country, the content of PM2.5 in the air is constantly increasing. Inhaled particles floating in the air will not only cause environmental impacts, such as urban climate change and decline in traffic conditions, but also endanger human health. From the perspective of people's generally accepted urban life and special attention to health, the goal of urban development is to start with the initial pursuit of maximizing economic benefits in a high-quality urban environment and then continue to move towards creating a friendly city. However, smoggy weather is now an obstacle to the development of environmentally friendly cities. China belongs to the heavily polluted area of PM2.5 in the world, so we need to monitor and analyze the environment of healthy streets in cities. At present, more and more sensors are deployed on mobile terminals. A large number of ordinary users use these mobile devices to cooperate with the Internet to realize the distribution of perception tasks and the collection of perception data to complete large-scale and complex social perception tasks [1]. This technology is called crowd sensing.

1.2. Significance. Exploring the planning and design methods for improving urban ventilation and improving the quality of the urban air environment are the living needs of every urban resident and a good guarantee for providing citizens with a healthy living environment. In view of the many hazards of PM2.5 in the environment, transportation, and health, according to the urban industrial layout and urban spatial pattern, it is analyzed that PM2.5 frequently 
occurs in streets and high-density streets, and the residence time of citizens in the street is controlled. At the planning and design level, the hidden dangers that hinder the spread of pollutants are eliminated in advance. In addition, this research is also an inevitable demand to enhance the image of the city, and it is of great significance for the construction of an ecological city and an environment-friendly city.

1.3. Related Work. By making use of mobile devices and smart phone users worldwide, mobile crowd sensing technology can realize a variety of novel applications. Although this paradigm is very useful, it involves gathering detailed information from sensors and their carriers (i.e., participants) in the task management process (including sensor recruitment and task assignment). Such information may compromise the privacy of participants in various ways by identifying or disclosing sensitive attributes, thereby increasing vulnerability and subsequently reducing participation. Pournajaf et al. proposed the use of management methods in crowd sensing to manage different tasks and related assessments of the threats to participants' privacy when personal information is disclosed. They will also outline how to use privacy mechanisms in existing sensing applications to deal with these threats. Finally, they discussed the continuing challenges faced by participant privacy protection methods in the task management process [2]. However, they only used crowd sensing technology for task management and did not conduct relevant research and explanations on urban street environment management. Kulkarni's research discusses a conceptual structural health monitoring (SHM) system for monitoring fatigue damage. The first part of the research is related to the prognosis of damage. A method is proposed to use data from the precrack damage monitoring system in a probabilistic sense. The issues related to quantification of damage, the law of damage evolution, and the numerical evaluation of macroscopic crack germination probability are discussed. The second part of the research involves quantifying the impact of imperfect inspections during the macroscopic crack growth process. The possibility that there is an undetected critical crack is the number of interest. For two-dimensional cracks, an analytical expression of this quantity is derived. A Monte Carlo simulation method is also proposed to quantify the impact of imperfect inspections. Numerical results give examples of two surface cracks with different geometric shapes [3]. However, Kulkarni's research is only to monitor the bridge structure and does not use mobile group intelligence sensing technology to monitor the health of the street. Adam B's structural health monitoring (SHM) using the wireless sensor network (WSN) can reduce the costs related to the installation and maintenance of the SHM system, thus winning research interest. The SHM system has been used to monitor critical infrastructure such as bridges, high-rise buildings, and stadiums and has the potential to improve structural life and improve public safety. The high data collection rate of WSN for SHM presents unique network design challenges. Adam gave a comprehensive overview of SHM using WSN, an overview of algorithms for damage detection and location, and an overview of network design challenges and future research directions. Comparing and discussing solutions to network design issues, such as scalability, time synchronization, sensor placement, and data processing, the survey also outlines the test platform and actual deployment of WSN for SH [4]. However, they only used wireless sensors to monitor various infrastructure and facilities and did not conduct relevant research on mobile positioning technology.

1.4. Innovation. At present, there are many research results on environmental monitoring in foreign countries, and many domestic researches on environmental monitoring, but most of them are macro studies. There are very few research results on the microscopic environmental monitoring of a specific city. This paper takes the street environment monitoring in some areas of Shanghai as the research object and proposes the main technologies used to realize a PM2.5 measurement system with high accuracy and corresponding speed. This study first introduces the convolutional neural network model used to measure PM2.5 concentration through images, the Resnet-50 training model that uses image features to measure the concentration of PM2.5, and the machine learning platforms TensorFlow and TFLearm; then the use of crowd sensing to monitor the environment data is fused, and finally the adaptive weighted data fusion method is introduced. All these key technologies provide theoretical and technical support for the realization of the subsequent system.

\section{Group Intelligence Perception Urban Healthy Street Monitoring Method}

2.1. Crowd Sensing. With the development of mobile crowd sensing networks, different perception network architectures have emerged according to different application scenarios [5]. This paper presents a basic mobile swarm perception network system architecture [6]. As shown in Figure 1, the mobile crowd sensing network is divided into three layers using the data transmission process, namely, the mobile perception layer, the transmission layer, and the service processing application layer. On the basis of this architecture, it can be extended according to different scenarios. For example, a privacy protection layer can be added to the mobile perception layer. All data perceived by the user is not directly uploaded to the service platform but sent to the server after processing on the mobile smart end [7]. In order to improve the operating efficiency of the system and ensure the real-time performance of the data, a special data channel layer can be added to the transmission layer to give priority to the rapid transmission of special data to the server and so on [8].

(1) Mobile sensing layer is the most basic and most important layer in the mobile group intelligence sensing network. This layer is composed of smart devices in people's hands. At the beginning of the sensing task, the central server publishes the task on the mobile sensing smart device. People use the built-in sensors in the mobile smart device, 


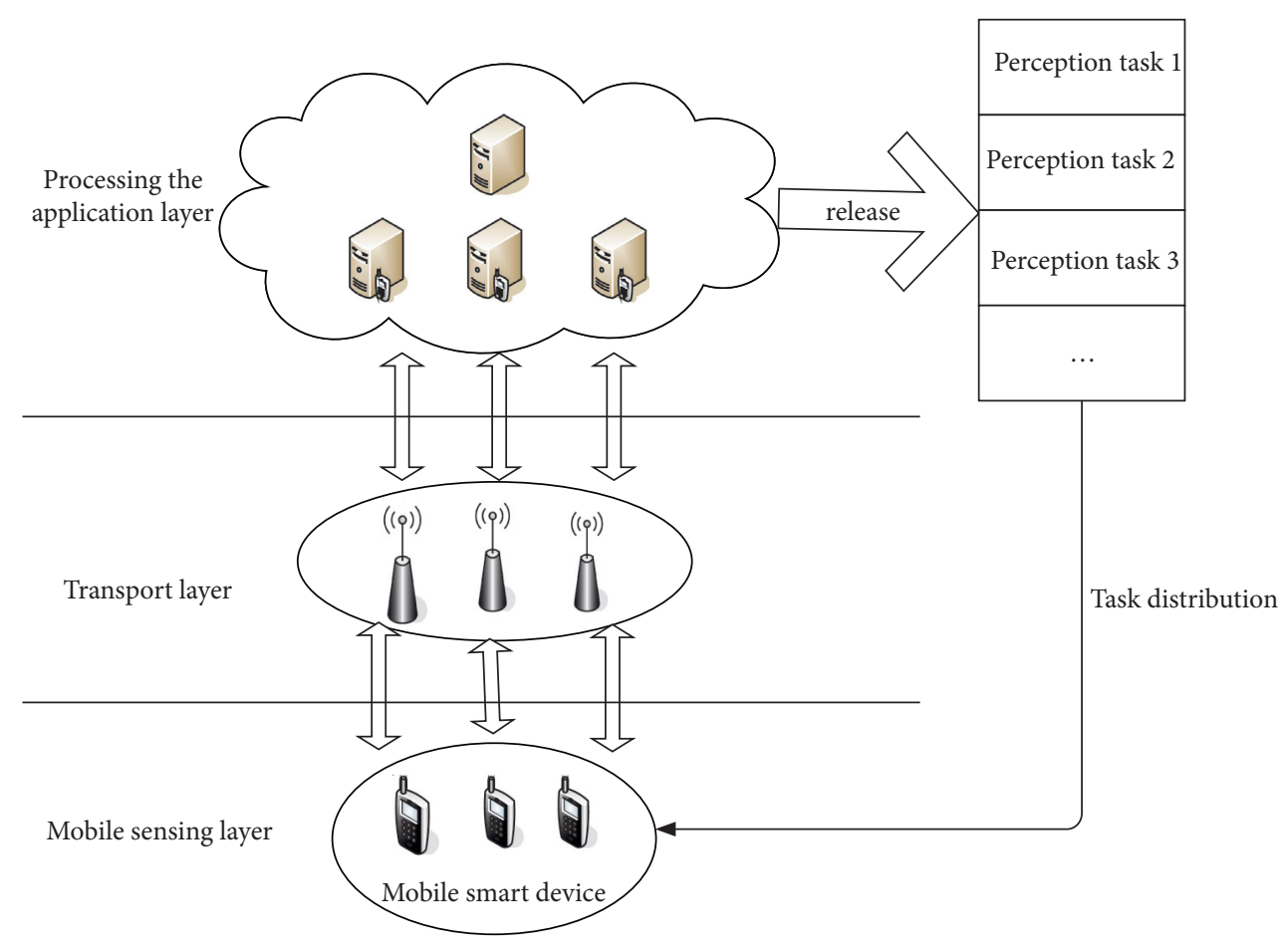

FIgURE 1: Three-tier architecture model of the mobile crowd sensing network.

such as camera, light sensor, GPS, accelerator, compass, gyroscope, microphone, and proximity sensor for data collection. The mobile sensing layer is the most important data collection source layer in the mobile swarm intelligence network [9]. (2) Transport layer provides a guarantee for data transmission from the mobile sensing layer to the central server. This layer can use mobile network infrastructure, such as $5 \mathrm{G}$ network, WiFi network, and other networks, for data transmission and can also use network nodes built by other users around for transmission. The efficiency of this layer mainly depends on the coverage of the network around the mobile terminal and the network transmission. With the rapid popularization of mobile networks and the improvement of transmission speed, the transmission layer becomes more and more reliable and real-time [10]. (3) Service processing application layer is equivalent to the human brain in the mobile swarm perception network. After receiving the data from the transport layer, the service processing application layer needs to store and calculate the data to complete the task of application publishing [11]. The performance of this layer depends on the configuration of the hardware and the implementation of the algorithm. In the crowd sensing network, the mobile perception layer is composed of a large number of mobile smart devices. Many participants are not professional, and the standards of smart-sensing devices are not uniform. Therefore, there are a lot of inaccurate, incomplete, and not timely data and other issues. In this layer, the data needs to be corrected to obtain valuable data for the perception task [12]. The above three layers are the simplified architecture of the mobile crowd sensing system. In reality, the complexity of the system architecture is far more than that. It can be extended on this basis; for example, a security privacy layer can be added to the mobile perception layer [13]. The working process of the system is mainly divided into the following five steps: (1) the mobile crowd sensing platform divides the perception task into multiple perception subtasks and then distributes these perception subtasks to the mobile intelligence terminal and sets the mobile crowd sensing incentive mechanism to motivate users to participate. (2) After the participants get the perception task, they will decide whether to accept the perception task according to their own participation costs [14]. (3) Participants use the sensors of the mobile smart device to obtain the perception data after accepting the perception task and then transmit the perception data to the perception service platform. (4) The perception service platform will evaluate the data submitted by the participants and then pay the participants [15]. (5) The perception service platform receives the data transmitted by the mobile smart terminal and analyzes it after decrypt and other processing. Using these data, a variety of application systems can be constructed, such as infrastructure service systems and intelligent transportation systems [16].

The performance of the sensor network largely depends on the number of sensors in the network. Insufficient node coverage will affect the monitoring quality of the target area, and adding nodes will increase the cost of network installation and maintenance [17]. Crowd sensing is essentially a sensor network, but it has unique characteristics and advantages, as shown in Table 1.

Although crowd sensing has many advantages, there are some other problems that need to be solved in the process of crowd sensing: (1) the credibility of the data provided by the 
TABLE 1: Comparison of crowd sensing and wireless sensor network.

\begin{tabular}{lcc}
\hline & Wireless sensor network & Crowd sensing \\
\hline Owner & Company or government & Personal \\
Sensor energy & Limited & Can be added \\
Sensor mobility & Low & High \\
Perceived content & Only physical phenomena & Physical phenomena and human daily life \\
Data generator & Some specific environment & Human beings and their surroundings \\
Sensor & Large number of small devices & Existing equipment \\
Scalability & Low & High \\
Cost & High & Low \\
\hline
\end{tabular}

user. Another security issue of group intelligence is the reliability of uploaded data. Since data is reported by participants, they may be falsified. Therefore, this raises the question of data credibility, which conflicts with privacy issues. This is because if the identity of the participant is not disclosed, the data reported or forged by the participant cannot be identified and eliminated. That is to say, if the crowd sensing provides a completely anonymous service to the participant, it is difficult to ensure that the data reported by the participant is available. (2) Reliability: the information team recognizes the processing and transmission of data. The data collected by mobile group intelligence is usually noisy or nonstandard data quality and cannot be directly passed to the perception group intelligence application, so the initially collected perception data must be processed. On the other hand, different users, devices, and sensors collect perceptual data that need to be analyzed and combined to obtain useful information. After completing the data processing process, the user must upload the perception data to the service platform through the available network. Therefore, in order to facilitate the sending of large amounts of data, it is necessary to design an effective data transmission method in combination with actual scenarios [18]. (3) Resource allocation in the information group perception system: the promotion of crowd sensing real-time detection network also faces resource constraints, such as bandwidth, frequency range, and power. In order to complete a specific perception task, it is necessary to model and predict resource allocation, but due to the uncertainty of the number of users, the crowd sensing system makes the modeling process more difficult [19].

\subsection{Data Fusion of Urban Street Health Monitoring Based on} the Distribution Map Method. Due to the limitation of the performance of the sensor itself, the strong interference in the road environment, and the failure of the test equipment itself, when measuring street environmental pollution data, it is unavoidable that large measurement errors will occur, that is, negligence errors [20]. Negligence errors seriously affect the consistency of the measured data, so the consistency of the data is checked before data fusion, and the ignorance errors are eliminated, which not only lays the foundation for subsequent data fusion processing but also improves the reliability and accuracy of data fusion [21]. For the data obtained by the street environmental pollution monitoring and collection system, the distribution map method is used to check the consistency [22, 23]. The algorithm of the distribution map method is as follows: first, assume that the $\mathrm{K}$ measurement results obtained by a certain sensor are sorted in ascending order to obtain the sequence of the following formula:

$$
S_{1}, S_{2}, \ldots, S_{K}
$$

Among them, $S_{1}$ is called the lower limit of the measurement, and $S_{K}$ is called the upper limit. The median value is shown in the following formulas:

$$
\begin{aligned}
& S_{A}=S_{K+1 / 2} \quad K=2 i+1, \\
& S_{A}=\frac{S_{K+1 / 2}+S_{K / 2}}{2} \quad K=2 i .
\end{aligned}
$$

The upper quartile $Z_{\mu}$ is the median of interval $\left[S_{A}, S_{K}\right]$, and the lower quartile $Z_{l}$ is the median of interval $\left[S_{l}, S_{A}\right]$. The dispersion of quartiles can be expressed by the following formula:

$$
d Z=Z_{\mu}-Z_{l}
$$

Then the judgment interval of valid data is $[c 1, c 2]$, as shown in the following formulas:

$$
\begin{aligned}
& c 1=Z_{l}-\frac{\alpha}{2} d Z, \\
& c 2=Z_{\mu}+\frac{\alpha}{2} d Z .
\end{aligned}
$$

2.3. Street Environmental Pollution Data Fusion Based on Adaptive Weighting. The adaptive weighted data fusion algorithm has different weights for different groups of measurement data. Under the optimal condition of the smallest total mean square error, the corresponding weights are found in an adaptive manner according to each group of measurement data to make the fusion of the latter value reaches the optimal rate [24]. The algorithm structure diagram is shown in Figure 2.

There is a monitoring system in which there are $n$ sensors to monitor and sample a certain research object. The monitoring data are $Y_{1}, Y_{2}, \ldots, Y_{n}$, respectively, and the variance of each sensor node is $\sigma_{1}^{2}, \sigma_{2}^{2}, \ldots, \sigma_{n}^{2}$, respectively. The final fusion value of the multisensor data should be as shown in the following formula: 


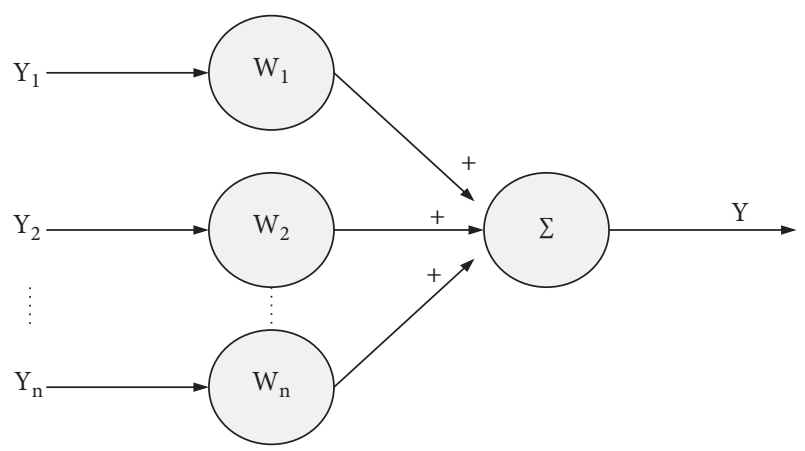

FIgURE 2: Adaptive weighted data fusion model.

$$
\widehat{Y}=\sum_{k=1}^{n} W_{k} X_{k}
$$

So the total mean square error is shown in the following formula:

$$
\sigma^{2}=\sum_{k=1}^{n} W_{k} \sigma_{k}^{2}
$$

According to the extreme value method of multivariate function, the weighting factor when the mean square error is the smallest can be obtained as in the following formula:

$$
W_{k}=\frac{1}{\sigma_{k}^{2} \sum_{k=1}^{n} 1 / \sigma_{k}^{2}} .
$$

At this time, $\sigma^{2}$ is the minimum value and can be expressed as shown in the following formula:

$$
\sigma_{\min }^{2}=\frac{1}{\sum_{k=1}^{n} \sigma_{k}^{2}} .
$$

The parameter value of the measured object is an objective constant, so it can be estimated based on the arithmetic average of the existing monitoring data. Assuming that the k-th group of sensors performs $m$ measurements, then there is the following formula:

$$
\bar{Y}_{k}(m)=\frac{1}{m} \sum_{i=1}^{m} Y_{k}(i) .
$$

The estimated value can be obtained as shown in the following formula:

$$
\widehat{\bar{Y}}=\sum_{k=1}^{n} W_{k} \bar{Y}_{k}(m) .
$$

The total mean square error can be expressed by the following formula:

$$
\bar{\sigma}^{2}=\frac{1}{m} \sum_{k=1}^{n} W_{k}^{2} \sigma_{k}^{2} .
$$

From formulas (10) and (13), we can deduce the following formula:

$$
\bar{\sigma}^{2}=\frac{1}{m \sum_{k=1}^{n} \sigma_{k}^{2}}=\frac{\sigma_{\min }^{2}}{m} .
$$

Obviously, $\bar{\sigma}^{2}<\sigma_{\min }^{2}$ and, as $k$ increases, $\bar{\sigma}^{2}$ gradually decreases.

Because the adaptive algorithm can adaptively select a more suitable weighting factor according to different measurement values to make the fused data more accurate, the second layer of data fusion in this system adopts an adaptive weighted data fusion algorithm.

2.4. Pm2.5 Sensor. PM2.5 usually refers to particles with a diameter of no more than $2.59 \mathrm{~m}$. PM2.5 sensor is also called dust sensor, which can be used to detect the dust concentration in the air around us, that is, the value of PM2.5. In aerodynamics, dust with a diameter of less than 10 microns that can enter the alveolar area is usually also called respiratory dust [25]. Most of the dust particles with a diameter of more than 10 microns are deposited by impact, and most of them are deposited in the nasopharynx when inhaled by the human body, while dust below 10 microns can enter the deep part of the respiratory tract [26]. The dust deposited in the alveoli is mostly dust below 5 microns. PM2.5 fine particles are small in diameter, are suspended in the atmosphere for a long time, spread and spread far, and usually contain a large number of toxic and harmful substances, so they have a greater impact on human health. PM2.5 can enter the lungs and blood. Carrying germs can be very harmful to the human body. There are three principles for PM2.5 monitoring: weighing method $\beta$, ray method, and light scattering method. For sensors, the light scattering method is the real method that can be used to measure PM2.5 [27]. The light scattering method (MIE meter type) has two typical types: laser and infrared light. The laser is based on infrared light and uses a more enhanced beam, namely, laser, plus a "fan" design unique air duct, which makes up for the application. There are some shortcomings of the infrared principle (air flow is required, and the accuracy is low) [28]. The structure and circuit of the infrared dust sensor are relatively simple. The light source is an infrared LED light source, and the air inlet and outlet mainly rely on resistance heating to obtain the hot air flow, and the output is high when particles pass. The structure and circuit of the laser sensor are relatively complicated. The light source is a laser diode. The sampled air is pushed by a fan or blower and passed through a complicatedly designed air duct for detection.

\section{Design Experiment of the Smart Perception Urban Healthy Street Monitoring System Based on Mobile Positioning}

3.1. Overall System Framework. According to the system designed in this paper, the content of PM2.5 has great regional differences. Therefore, PM2.5 monitoring requires not only real-time connection to the network to transmit data, but also a real-time location of the user. Looking for chips on the market, Anxin-A7 can meet this requirement. 
The module is designed to take these issues into consideration. Therefore, the module can use positioning and GSM/ GPRS functions at the same time, which saves resources and reduces the time for switching back and forth between various functions. This chip can carry out $2 \mathrm{G}$ data interconnection, although the speed is not very fast, but it can already meet our needs, and the price is relatively low, so this chip is used. The GPS positioning function of this module is enabled by external voltage, high-level enabler, or through AT command. This system uses high-level enabler. The overall framework is shown in Figure 3:

This system is divided into four modules: related sensor circuit module, mobile positioning module, power supply module, and real-time monitoring module. The mobile positioning module is responsible for tracking and positioning the monitored street area, and the relevant sensor circuit module is responsible for monitoring PM2.5 in the environment. The content is transmitted to the sensor through infrared or laser, the real-time monitoring module is responsible for monitoring whether the PM2.5 content in the air exceeds the standard, and the power module is responsible for providing power and signals for this system.

3.2. Image Set Based on Crowd Sensing. Machine learning needs to be supported by a large amount of data, because the corresponding image set is not used on the public data set. In order to collect enough feature images for training, we adopted the method of group intelligence and made use of laboratory resources to launch a competition to shoot the sky. The top students who took the most photos every day were given cash rewards, and a collection was developed. The image APP is for students to use, which arouses the enthusiasm of students to participate in scientific research activities. After this event, a total of more than 50,000 images were collected, including more than 30,000 valid images. The upper part of the image is the sky and the lower part is the building. It is similar to the data set environment picture in Figure 4.

While uploading, you can use the location information to reverse the measured value of the PM2.5 concentration of the nearest air detection site, and to label the uploaded image. Table 2 marks the image into 6 categories according to the PM2.5 concentration of the image.

3.3. Selection of Training Set and Test Set. The training image set we selected comes from our daily shooting and the buildings and sky images obtained in the Baidu gallery. We choose the more mature convolutional neural network model Resnet-50 as the training model. Although Resnet-50 may not be very effective for object feature extraction and target indicators, it is very suitable for experiments with fewer parameters, because Resnet-50 has a relatively high calculation accuracy and a small amount of calculation. It is currently a widely used deep learning training model. The input data first undergoes a $1 \times 1$ convolution for dimensionality reduction, then a $3 \times 3$ convolution kernel for convolution, and finally a $1 \times 1$ convolution kernel for dimensionality improvement. This can reduce training

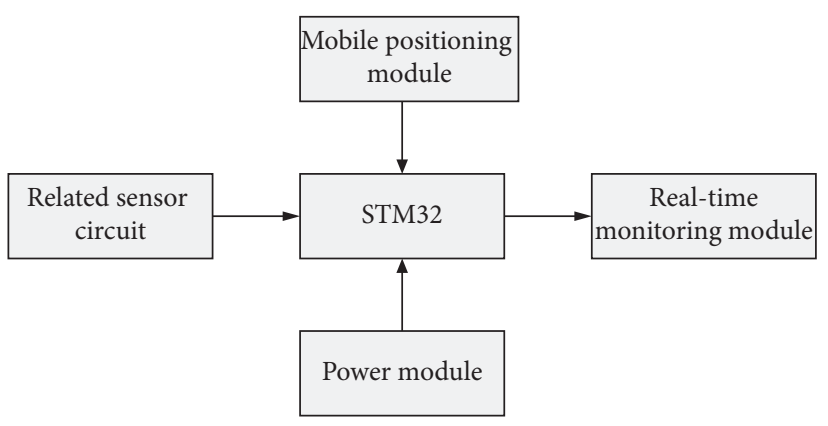

FIgURE 3: The overall framework of the system.

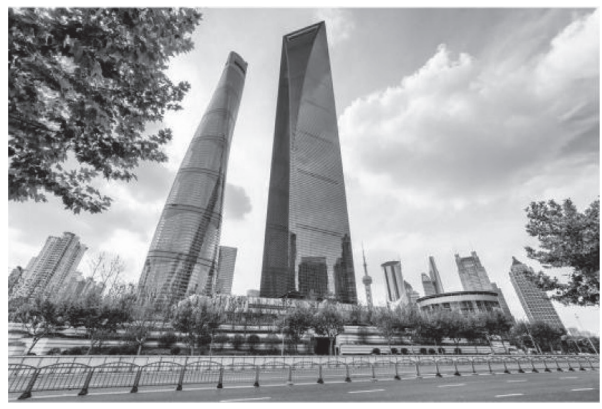

Figure 4: Collected sky images.

TABle 2: Classification of image PM2.5 concentration levels.

\begin{tabular}{lc}
\hline Image level & pm2.5 concentration \\
\hline Level 0 & $0-50$ \\
Level 1 & $51-100$ \\
Level 2 & $101-150$ \\
Level 3 & $151-200$ \\
Level 4 & $201-300$ \\
Level 5 & $301-500$ \\
\hline
\end{tabular}

parameters and reduce training time while maintaining the complexity of the network. Figure 5 is a residual module of Restnet-50, which is composed of three layers of convolution and an identity map.

\subsection{Normalized Processing of Street Environment Monitoring} Data. If you want to predict PM2.5 correctly, you must determine whether there is a correlation between PM2.5 and each gas in the model. At my current stage, most domestic measures of air pollution use the AQI index, and the AQI index is related to six monitoring indicators in the air, namely, $\mathrm{SO} 2, \mathrm{NO} 2, \mathrm{CO}, \mathrm{O} 3, \mathrm{PM} 10$, and PM2.5. Because the actual measured data cannot reach the level of training the BP neural network, we can only call the relevant data of the National Meteorological Network. This article selects the data of Shanghai from 2018 to 2020 . The two-year data is selected because the amount of data is too small to achieve the effect of training, and if the time is too long, it will cause a lot of training time, so this paper uses more than two years of data to complete the prediction model design. First of all, a 


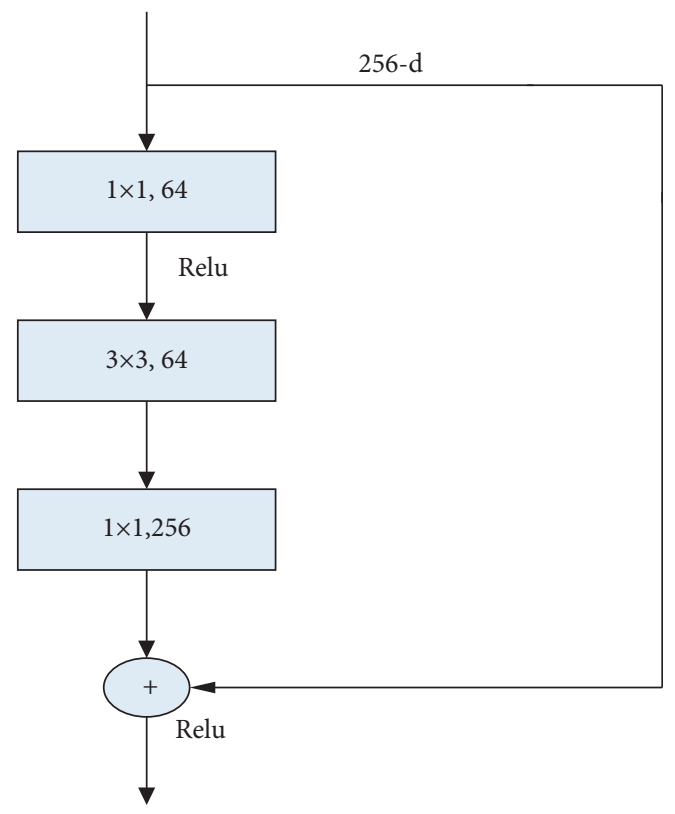

FIgURE 5: Resnet-50's bottleneck residual module.

line drawing of all PM2.5 historical data collected in Shanghai is shown in Figure 6.

It can be seen from Figure 6 that the PM2.5 data is messy. If you only want to use the historical PM2.5 data to do the fitting to get the desired predicted value, it is not realistic. This requires the involvement of other relevant data. At this stage, the AQI index is generally used for the evaluation of air quality, and under normal circumstances, the meteorological station will generally give the value of PM10, NO2, $\mathrm{CO}, \mathrm{SO} 2$, and PM2.5 when the AQI index is given. We selected some historical weather data of Shanghai on the China Meteorological website and organized them into a data set. Some of the data is shown in Table 3.

After having the data, we must first determine whether there is an internal correlation between the various indicators. If there is no connection between the data, it will not be of any help to the PM2.5 prediction work. First look at whether the AQI index and the PM2.5 index are related or not. If the AQI index is related to the PM2.5 index, then other gases given together should also have a certain relationship. In this paper, a corresponding linear fitting is made on the AQI index and PM2.5 index, and the fitting diagram is shown in Figure 7.

It can be seen from the fitting graph that the AQI index and PM2.5 are highly correlated, roughly a linear correlation. However, if each is analyzed in this way, it will consume a lot of time and cost. In order to quickly determine whether there is a correlation between these AQI indicators, we analyze the overall AQI indicators and obtain the correlation coefficient table as shown in Table 4.

It can be seen from Table 4 that there is a certain correlation between the various indicators related to air quality. Among them, PM10, NO, CO, SO2 and PM2.5 are positively correlated, and $\mathrm{O} 3$ and PM2.5 are negatively correlated. The results of this analysis are consistent with the relevant literature. At the same time, through reading the literature, we

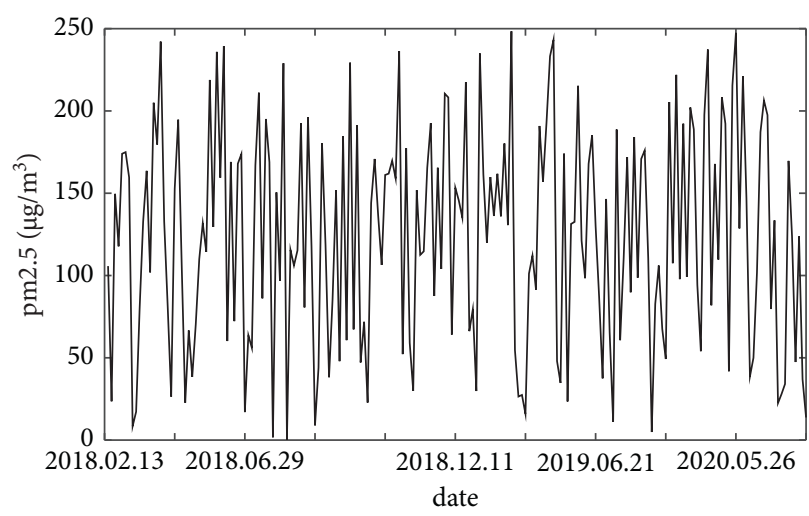

FIgURE 6: Shanghai PM2.5 data graph from 2018 to 2020.

Table 3: Air quality data set in some areas of Shanghai.

\begin{tabular}{lcccccccc}
\hline Date & AQI & PM2.5 & PM10 & NO2 & SO2 & O3 & CO & TEMP \\
\hline 2020.04 .21 & 147 & 123 & 174 & 40 & 35 & 22 & 1.25 & 18 \\
2020.04 .22 & 123 & 114 & 148 & 30 & 28 & 9 & 1.14 & 17 \\
2020.04 .23 & 112 & 105 & 132 & 25 & 23 & 15 & 0.67 & 20 \\
2020.04 .24 & 73 & 62 & 85 & 35 & 32 & 12 & 0.97 & 26 \\
2020.04 .25 & 204 & 189 & 198 & 41 & 43 & 25 & 1.68 & 24
\end{tabular}

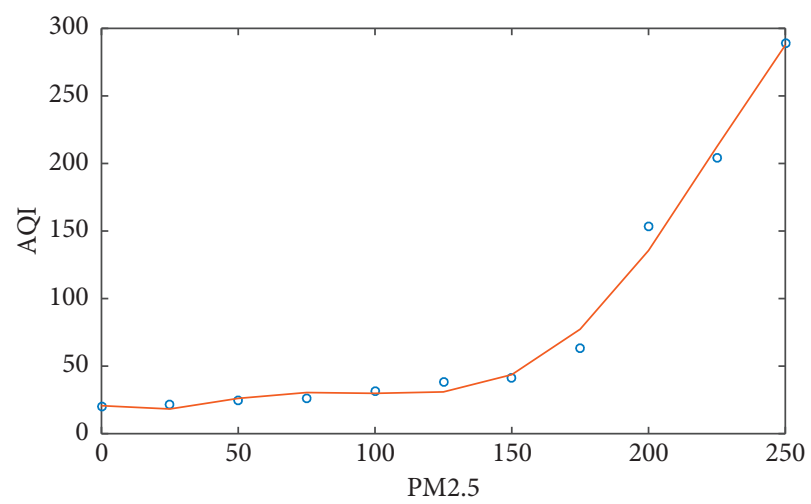

FIgURE 7: AQI/PM2.5 fitting diagram.

TABLE 4: Correlation coefficient table.

\begin{tabular}{lcccccc}
\hline $\begin{array}{l}\text { Correlation } \\
\text { coefficient }\end{array}$ & PM2.5 & PM10 & NO2 & SO2 & O3 & CO \\
\hline PM2.5 & 1.00 & 0.95 & 0.54 & 0.45 & -0.32 & 0.76 \\
PM10 & 0.95 & 1.00 & 0.79 & 0.67 & 0.03 & 0.52 \\
NO2 & 0.54 & 0.79 & 1.00 & 0.69 & -0.45 & 0.47 \\
SO2 & 0.45 & 0.67 & 0.69 & 1.00 & 0.41 & 0.52 \\
O3 & -0.32 & 0.03 & -0.45 & 0.41 & 1.00 & -0.88 \\
CO & 0.76 & 0.52 & 0.47 & 0.52 & -0.88 & 1.00 \\
\hline
\end{tabular}

know that temperature and PM2.5 have a negative correlation. The specific reason is that the lower the temperature is, the closer the dust and particulate matter will be to the ground, and the worse the air quality will be. Through analysis, it can be known that there is a correlation between the various indicators of AQI, and a certain rule can be 
found by studying its data. Therefore, it can be seen that it is feasible to combine the historical data of the AQI index data with the historical data of PM2.5 for PM2.5 modeling and forecasting. Due to the gap between the counting child units and some other factors, the numerical gap between various data is relatively large. If this data is used to directly train the BP neural network, the larger data PM10, NO2, SO2, and PM2.5 prediction will occupy a larger weight. The values of $\mathrm{O} 3$ and temperature are in the middle of all data, and the weights assigned may be smaller. Because the value of $\mathrm{CO}$ is very small, it may be ignored in the final training model. This will cause the final result bias due to the weight problem and the data omission problem. In order to avoid the unevenness of the value, it is necessary to normalize the value. There are two forms of normalization. One is to change the number to a decimal between $(0,1)$, and the other is to express the dimensionality. The formula becomes a dimensionless expression. This article adopts the first form. The processed values are shown in Table 5.

\section{System Function Test and Satisfaction Survey}

4.1. Accuracy Test of Pm2.5 Monitoring in Street Environment. The system performance test is divided into two parts: the accuracy test of the monitoring module and the system stress test. In the accuracy test module, the accuracy of the model based on image monitoring of PM2.5 concentration and the accuracy of the model for monitoring PM2.5 concentration of weather characteristics are tested respectively. The performance test module tests the response time of the system query interface in the cluster and the response time of the stand-alone service. From the results of the system display, we can see that the accuracy of image-based monitoring of PM2.5 concentration is basically above $90 \%$. In the case of relatively low PM2.5 concentration, the model monitoring is more accurate, while in the case of relatively high PM2.5 concentration, the accuracy of model monitoring has decreased. We analyzed the notion that it may be caused by insufficient training samples with relatively high PM2.5 concentration. In the future, we need to pay attention to collecting pictures with relatively high PM2.5 concentration and adding them to the training set.

4.2. Satisfaction Survey of Street Residents. After completing the overall design of the system and the analysis of the functional module requirements, we conducted a simulation experiment on the smart-sensing urban health street monitoring system based on the mobile positioning system in the next few months. We invited 102 community owners and street managers from a certain street in Shanghai. They come from different parts of the country. They are divided into three different age groups, and they have different educational backgrounds and personalities. Then, we analyzed the PM2.5 environment designed in this article. The monitoring system carried out a questionnaire satisfaction survey. A total of 102 questionnaires were sent out, 101 questionnaires were returned, 1 invalid questionnaire was removed, and 100 valid questionnaires remained. A
TABLE 5: Normalization of street environment monitoring data.

\begin{tabular}{ccccccc}
\hline PM2.5 & PM10 & NO2 & SO2 & O3 & CO & TEMP \\
\hline 0.13169 & 0.01475 & 0.16791 & 0.09478 & 0.14798 & 0.13675 & 0.263975 \\
0.23658 & 0.04197 & 0.26975 & 0.21478 & 0.32578 & 0.26478 & 0.147896 \\
0.36741 & 0.12478 & 0.23698 & 0.32697 & 0.19874 & 0.39156 & 0.174584 \\
0.45127 & 0.19164 & 0.46123 & 0.12369 & 0.36742 & 0.34972 & 0.269871 \\
\hline
\end{tabular}

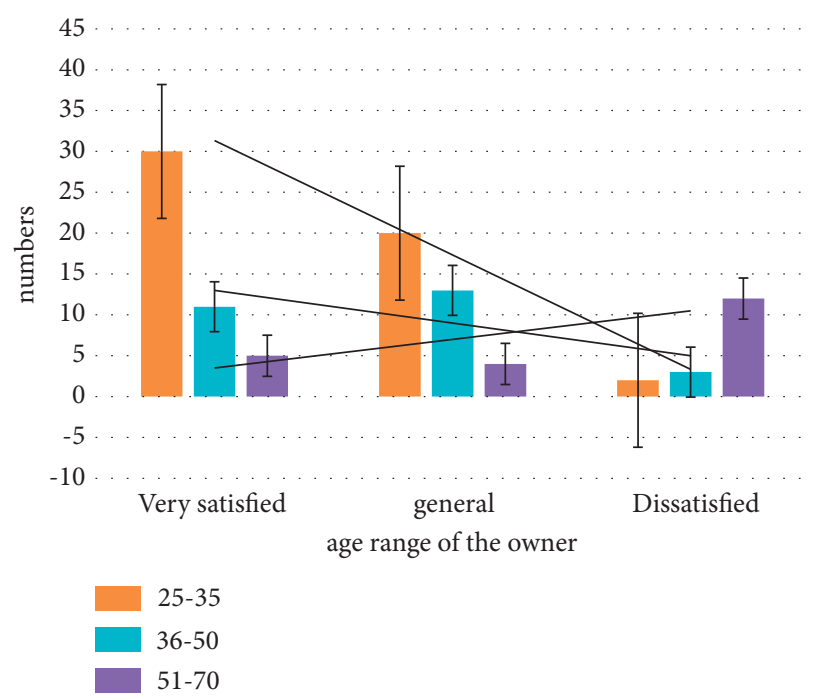

FIgURE 8: Survey form for satisfaction of street residents.

statistical description is carried out, and the result is shown in Figure 8.

It can be seen from Figure 8 that most young owners are satisfied with the design of the PM2.5 environmental monitoring system and believe that the monitoring system can supervise the health management of urban streets, allowing residents to understand the PM2.5 content in real time. However, a small number of elderly people are dissatisfied with the monitoring system designed this time. They believe that the urban street environmental health monitoring system designed this time is not practical. They believe that some more stringent vehicle restrictions should be introduced to reduce the air pollution.

\section{Conclusions}

At present, air quality is declining and haze weather is frequent, and citizens in many countries or regions are facing the threat of high particulate matter concentration. This paper proposes a fine-grained PM2.5 monitoring system based on the crowd sensing model. The system can provide citizens with a fine-grained PM2.5 concentration distribution map to help them learn the current local concentration of fine particles and take corresponding protective measures in time. At the same time, the entire system also provides new technical ideas and data foundations for studying environmental issues. The full text first introduces the idea, system structure, advantages, and disadvantages of the crowd sensing model. Considering its 
advantages and disadvantages comprehensively, a mobile collection scheme based on crowd sensing mode is proposed, which replaces the traditional fixed-point collection mode with a small number of high-precision sensors with a large number of low-cost sensor mobile collection methods and points out the corresponding advantages and technical difficulties. Due to the high cost of the system and limited resources, the current prototype system cannot truly achieve hundreds or thousands of crowd sensing. In this paper, the whole area is graphed, and the path planning function is designed to solve the specific mobile collection route, so that the data collection of the whole system is still efficient and accurate under the condition of limited resources. The environmental monitoring system test found that the accuracy rate of this system on the PM2.5 content in the monitoring pictures reached more than 90\%, which can meet the expectations of the developers.

\section{Data Availability}

No data were used to support this study.

\section{Conflicts of Interest}

The authors declare that they have no conflicts of interest.

\section{Acknowledgments}

This work was supported by the 2018 Youth Project for Humanities and Social Science Research of the Ministry of Education that funded the results of the "Construction of Shared Street Space System under the Guidance of "Urban Repair,"” Item no. 18YJCZH067.

\section{References}

[1] H. Lu, Q. Liu, X. Liu, and Y. Zhang, "A survey of semantic construction and application of satellite remote sensing images and data," Journal of Organizational and End User Computing, vol. 33, no. 6, 2021.

[2] L. Pournajaf, D. A. Garcia-Ulloa, L. Xiong, and V. Sunderam, "Participant privacy in mobile crowd sensing task management," Acm Sigmod Record, vol. 44, no. 4, pp. 23-34, 2016.

[3] S. S. Kulkarni and J. D. Achenbach, "Structural health monitoring and damage prognosis in fatigue," Structural Health Monitoring, vol. 7, no. 1, pp. 37-49, 2016.

[4] B. N. Adam, "Structural health monitoring using wireless sensor networks: a comprehensive survey," IEEE Communications Surveys \& Tutorials, vol. 19, no. 3, pp. 1403-1423, 2017.

[5] A. H. Ismail, Y. Mizushiri, Y. Mizushiri et al., "A novel automated construction method of signal fingerprint database for mobile robot wireless positioning system," International Journal of Automation Technology, vol. 11, no. 3, pp. 459-471, 2017.

[6] Y. Ji, A. Yamashita, and H. Asama, "Indoor positioning system based on camera sensor network for mobile robot localization in indoor environments," Journal of Institute of Control, Robotics and Systems, vol. 22, no. 11, pp. 952-959, 2016.

[7] S. G. Sukhpal and S. Arash, "Security-aware autonomic allocation of cloud resources: a model, research trends, and future directions," Journal of Organizational and End User Computing, vol. 32, no. 3, 2020.

[8] Y. Ji, A. Yamashita and H. Asama, "Indoor positioning system based on camera sensor network for mobile robot localization in indoor environments," Journal of Institute of Control, Robotics and Systems, vol. 22, no. 11, pp. 952-959, 2016.

[9] A. V. Teterev, "Rationale for the choice of a positioning system for mobile agricultural robot movement controlling," Agricultural Machinery and Technologies, vol. 14, no. 4, pp. 63-70, 2020.

[10] Q. Zou, W. Xia, and J. Zhang, "An indoor positioning system for mobile target tracking based on VLC and IMU fusion," Journal of Southeast University, vol. 34, no. 4, pp. 451-458, 2018.

[11] B. Nithiya and R. Eswari, "Efficient VM selection strategies in cloud datacenter using fuzzy soft set," Journal of Organizational and End User Computing, vol. 33, no. 5, 2021.

[12] Z. Xu, W. Yang, Z. Xiong, J. Wang, and G. Liu, “TPSense: a framework for event-reports trustworthiness evaluation in privacy-preserving vehicular crowdsensing systems," Journal of Signal Processing Systems, vol. 93, no. 2, pp. 209-219, 2021.

[13] C.-S. Wang, "An AR mobile navigation system integrating indoor positioning and content recommendation services," World Wide Web, vol. 22, no. 3, pp. 1241-1262, 2019.

[14] C. Peng and Z. Yu, "Modeling analysis for positioning error of mobile lidar based on multi-body system kinematics," Intelligent automation and soft computing, vol. 25, no. 4, pp. 827-834, 2019.

[15] C. Siebert, P. R. Destefano, and R. Widenhorn, "Teaching physics with a local positioning system," The Physics Teacher, vol. 57, no. 6, pp. 428-429, 2019.

[16] L.-H. Huang, K.-T. Shr, M.-H. Lin, and Y.-H. Huang, “A noise-robust convex-optimized positioning system based on code-aided RSS estimation and virtual base station transform," Journal of Signal Processing Systems, vol. 84, no. 3, pp. 309-323, 2016.

[17] L. He and L. He, "Beidou-GPS dual mode positioning of mobile communication equipment based on Android platform," Journal of Intelligent and Fuzzy Systems, vol. 40, no. 12, pp. 1-12, 2020.

[18] J. Wang, C. Guo, and L. Wu, "Gated recurrent unit with RSSIs from heterogeneous network for mobile positioning," Mobile Information Systems, vol. 2021, Article ID 6679398, 7 pages, 2021.

[19] W. Liu, Z. Li, S. Sun, R. Malekian, Z. Ma, and W. Li, "Improving positioning accuracy of the mobile laser scanning in GPS-denied environments: an experimental case study," IEEE Sensors Journal, vol. 19, no. 22, pp. 10753-10763, 2019.

[20] L. Yang and D. Xu, "Sliding mode control with PI-based saturation for nano-positioning," International Journal of Wireless and Mobile Computing, vol. 16, no. 3, pp. 204-210, 2019.

[21] B. Ergun, I. Sayim, C. Sahin, and N. Tok, "Real-time relative mobile target positioning using GPS-assisted stereo videogrammetry," Survey Review, vol. 50, no. 361, pp. 326-335, 2018.

[22] B. S. Noveck, "How to mobilize group intelligence," Nature, vol. 551, no. 7682, pp. 561-562, 2017.

[23] J. Chen, Y. Gao, C. Su et al., "Group intelligence-based decision making and its applications to traditional Chinese medical dysphagia rehabilitation treatment," Journal of Intelligent \& Fuzzy Systems: Applications in Engineering and Technology, vol. 31, no. 6, pp. 3181-3195, 2016.

[24] C. J. Huang, S. C. Chang, H. M. Chen, J.-H. Tseng, and S.-Y. Chien, "A group intelligence-based asynchronous 
argumentation learning-assistance platform," Interactive Learning Environments, vol. 24, no. 5-8, pp. 1408-1427, 2016.

[25] A. Riikonen, L. Jarvi, and E. Nikinmaa, "Environmental and crown related factors affecting street tree transpiration in Helsinki, Finland," Urban Ecosystems, vol. 19, no. 4, pp. 1-23, 2016.

[26] Z. Hao, T. Xu, Y. Wang, Y. Zong, S. Li, and H. Tang, "Study on the influence of meteorological conditions and the street side buildings on the pollutant dispersion in the street canyon," Building Simulation, vol. 9, no. 6, pp. 717-727, 2016.

[27] G. Y. Duan, H. L. Gong, H. H. Liu, Y. Q. Zhang, B. B. Chen, and K. C. Lei, "Monitoring and analysis of land subsidence along beijing-tianjin inter-city railway," Journal of the Indian Society of Remote Sensing, vol. 44, no. 6, pp. 915-931, 2016.

[28] N. Clements, M. P. Hannigan, and S. L. Miller, J. L. Peel and J. B. Milford, "Comparisons of urban and rural PM10-2.5 and PM2.5 mass concentrations and semi-volatile fractions in northeastern Colorado," Atmospheric Chemistry and Physics, vol. 16, no. 11, pp. 7469-7484, 2016. 